\title{
Research Paper: The Effect of 12 Weeks of Bariatric and Aerobic Exercises on Metabolic Syndrome Indices in Women
}

\author{
Sakine Sabzikar', Mehrdad Fathi ${ }^{* *}$, Teimour Darzabi ${ }^{3}$, Keyvan Hejazi $^{4} \mathbf{Q}$
}

1. Department of Physical Education and Sport Sciences, School of Sports Sciences, Bojnourd Branch, Islamic Azad University, Bojnurd, Iran. 2. Department of Physical Education and Sport Sciences, Faculty of Sport Sciences, Ferdowsi University of Mashhad, Mashhad, Iran 3. Department of Vocational Sciences, Faculty Technical of Shahid Montazeri, Technical and Vocational University, Mashhad, Iran. 4. Department of Physical Education and Sports Sciences, Toos Institute of Higher Education, Mashhad, Iran.

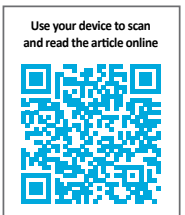

Citation Sabzikar S, Fathi M, Darzabi T, Hejazi K. The Effect of 12 Weeks of Bariatric and Aerobic Exercises on Metabolic Syndrome Indices in Women. Physical Treatments. 2018; 8(2):77-84. http://dx.doi.org/10.32598/ptj.8.2.77

http://dx.doi.org/10.32598/ptj.8.2.77

Article info:

Received: 28 Dec 2017

Accepted: 26 Mar 2018

Available Online: $01 \mathrm{Jul} 2018$
Keywords:

Exercise, Knee osteoarthritis, Insulin resistance, Lipid

\begin{abstract}
A B S T RA C T
Purpose: Osteoarthritis is a frequent complication in the middle-aged or older people. Besides pain and swelling, this disease causes deformity and difficulty in walking. The current study compared the effects of 12 weeks bariatric and aerobic exercises on the metabolic syndrome in women with knee osteoarthritis.

Methods: In this quasi-experimental study, 24 women with knee osteoarthritis were selected by convenience sampling method. They were randomly divided into two groups of the bariatric $(\mathrm{n}=12)$ and aerobic $(\mathrm{n}=12)$ exercises. The training included aerobic and bariatric exercises at $60 \%$ $65 \%$ rate of the heart rate reserve. The program included a 12 -week training, performed three times per week and each session lasted for 30 to 60 minutes. Intra- and inter-group comparisons were performed by dependent t-test and Analysis of Covariance (ANCOVA), respectively. The level of significance was set at $\mathrm{P}<0.05$.

Results: In both the aerobic and bariatric groups, 12 weeks of exercise program resulted in a significant reduction in weight, body mass index, serum glucose, insulin level, and insulin resistance index. Only in the aerobic exercise group, the concentrations of high-density lipoprotein, low-density lipoprotein, and total cholesterol significantly increased and decreased, respectively. The levels of triglyceride significantly decreased in both groups. Only in the aerobic exercise group, the maximum amount of oxygen consumption was significantly increased.

Conclusion: In women with knee osteoarthritis, 12 weeks of aerobic and bariatric exercises positively affect the levels of insulin resistance markers, lipid profiles, and max-oxygen consumption. Therefore, these two exercise programs can be used to maintain health of these people.
\end{abstract}

\footnotetext{
* Corresponding Author:

Mehrdad Fathi, PhD.

Address: Department of Physical Education and Sport Sciences, Faculty of Sport Sciences, Ferdowsi University of Mashhad, Mashhad, Iran. Phone: +98 (915) 2570058
}

E-mail:mfathei@um.ac.ir 


\section{Highlights}

- Aerobic and bariatric exercises have beneficial effects on the levels of insulin resistance markers, lipid profiles, and max-oxygen consumption.

- Both exercises can prevent the adverse effects of cardiovascular diseases and they can be considered as an essential part of the patient's lifestyle.

\section{Plain Language Summary}

Based on the study results, the aerobic and bariatric exercises can modulate the index of insulin resistance, maximal oxygen consumption, and lipid profile in women with knee osteoarthritis. Therefore, both types of exercises prevent the adverse effects of cardiovascular diseases and can be considered as an essential part of the patient's lifestyle. However, further research is needed to examine the changes in the markers of insulin resistance and lipid profile in women with knee osteoarthritis with different intensity of exercise and at different times.

\section{Introduction}

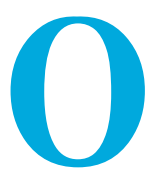

besity is a complex disorder resulting from an excessive accumulation of fat tissue. Obesity is among the negative factors affecting the health and longevity of the affected people. It is associated with numerous health problems such as osteoarthritis, metabolic syndrome, cardiovascular diseases, diabetes, hypertension, and high blood lipids [1]. Osteoarthritis or arthritis is a degenerative joint disease, which is also called progressive joint disease [2].

Osteoarthritis is a complex disorder identifiable by one or more risk factors such as gender, age, weight, height, Body Mass Index (BMI), and a history of osteoarthritis [3, 4]. Järvholm et al. on the basis of BMI, estimated the risk of severe arthritis in the knee joint or pelvis in individuals with normal- and over-weight people [5]. They concluded that the incidence of arthritis was directly correlated with increased BMI, even in the natural range or lower. In other words, a $5 \mathrm{~kg} / \mathrm{m}^{2}$ increase in BMI is associated with the doubled risk of severe knee osteoarthritis. However, another factor associated with osteoarthritis incidence is the prevalence of metabolic syndrome in obese women $[6,7]$.

Metabolic syndrome consists of a series of symptoms, including obesity, blood lipids, hypertension, glucose intolerance, and type 2 diabetes [8,9]. The prevalence of this syndrome is currently increasing in women compared with men [10]. Metabolic syndrome is detected when a person simultaneously has at least three of the following risk factors: a waist circumference of $\geq 102 \mathrm{~cm}$, a triglyceride level of $\geq 150 \mathrm{mg} / \mathrm{dL}$, a fasting blood glucose of $\geq 110 \mathrm{mg} / \mathrm{dL}$, and High-Density Lipoprotein (HDL) of $\leq 40 \mathrm{mg} / \mathrm{dL}$ [11]. Therefore, increasing insulin levels in response to insulin resistance plays an important role in the development of this disease [12].

Insulin resistance is defined as a defect in glucose response to a specific amount of insulin. Insulin levels increase in circulation to maintain glucose levels to compensate for this deficiency in many of these patients [13]. There is a relationship between insulin resistance and coronary heart disease $[14,15]$. In this regard, Pyörälä et al. found a significant relationship between hyperinsulinemia and coronary artery disease [16]. Exercise and physical activity help eliminating insulin resistance. One of the training that can help improve insulin resistance is bariatric training.

The bariatric training is inspired by a Table of 6 houses in space or on the ground, to implement the coach exercises by moving the hands and feet on the Table houses. There are contradictory data on the effect of exercise and proper nutrition on controlling the cardiovascular risk factors, metabolic disorders, increased prevalence of glucose intolerance, diabetes, lipid disorders, as well as the positive effect of a regular exercise program on the improvement and regulation of endocrine status $[17,18]$.

Bruno et al. examined the effect of three months of aerobic exercises on 38 women [19]. They concluded that insulin levels and insulin resistance index significantly decreased in the experimental group, while these variables increased in the control group. Lin et al. explored the effect of 12 weeks of exercise (30-minute sessions, three sessions per week) on the Quality of Life (QoL) and insulin sensitivity in 17 men [20]. They concluded that the level of insulin 
sensitivity increased; however, the amount of glucose and insulin resistance significantly decreased and depression and QoL were improved.

Weight loss is a non-pharmacological intervention for obesity, especially the abdominal type. Diet, physical activity, and pharmacotherapy are among other treatment methods. Given the prevalence of osteoarthritis as one of the musculoskeletal diseases in the Western countries, it is estimated that the risk of this disease in men's and women's thighs are about $18.5 \%$ and $28.6 \%$, respectively. The incidence rate of osteoarthritis is approximately $45 \%$ [6]. More than $40 \%$ of the Iranian population over the age of 70 suffer from knee arthritis [21]. In the United States, nearly 20 million people have knee arthritis [22].

This disabling disease is associated with reduced QoL. The prevalence of this disease is rising with increasing life expectancy. Therefore, the use of preventive interventions to reduce the risk of developing knee arthritis is important. This is because it not only impacts the QoL of patients but also reduces the economic burden of this disorder on the patients' family and society. Therefore, the current study compared the effects of bariatric and aerobic exercises on metabolic syndrome indices in women with knee osteoarthritis.

\section{Materials and Methods}

\section{Subjects}

This was a quasi-experimental study with a pre-test- Pretest design, conducted on the two groups of people performing aerobic $(n=12)$, and bariatric $(n=12)$ exercises in Shirvan City, Iran. The study participants comprised of 24 middle-aged women (30-45 years old) with premature knee osteoarthritis. They gathered together for the first time at the Shirvan Azad University to express their consent to participate in the study. Then, the study participants were given explanations of the research procedure.

The convenience random sampling method was applied to select the study participants. First, the subjects became familiar with the nature and manner of collaborating in the research. The inclusion criteria were women with chronic knee pain for at least 3 months, achieving functional levels of 1 and 3 (based on clinical and radiological signs), lack of pain in the acute phase of disease, lack of intra-articular injection of medications for the last 3 months, being menopause, lacking histories of trauma, injury or surgery, lower limb fracture, joint threatening diseases (osteonecrosis, diabetes, osteoporosis, rheumatoid arthritis, neuromuscular diseases, vascular collagen disease, psoriatic arthritis, and arthritis caused by gout and pseudogout), lacking long-term use of drug affecting the musculoskeletal system, lacking substance dependence. All of these cases were reviewed by an orthopedic specialist. Other inclusion criteria were no participation in any exercise programs for at least two months before the study and having a BMI of over $30 \mathrm{~kg} / \mathrm{m}^{2}$.

The exclusion criteria were the existence of cardiovascular diseases, diabetes, hypertension, and absence from two consecutive sessions or three non-consecutive sessions of the exercise program. In total, 30 volunteers completed a Short Form Survey Instrument (SF-36), regarding bio-psychological status and QoL (including daily physical activities, the occurrence of physical activity-related problems). After analyzing the data obtained from the questionnaire, 24 subjects were selected to participate in the study. The subjects participated in the research voluntarily and provided signed informed consent forms.

\section{Body composition}

To assess the body composition, the subjects' heights were measured using SECA (Germany) (with a sensitivity of 5 $\mathrm{mm}$ ). Also, the body fat percentage and weight were determined using a biomechanical impedance device (In Body72 , South Korea). BMI $\left(\mathrm{kg} / \mathrm{m}^{2}\right)$ was obtained by dividing body weight by squared height $(\mathrm{m})$. All of these measurements were performed when the subjects fasted for 4 hours before the test, and their bladders were emptied.

\section{Blood samples}

The blood samples were collected in two stages from the vein of the left hand of each subject in sitting position and resting position 48 hours before the initiation of training and then 48 hours after the last training session. All samples were obtained after 10 to 12 hours of fasting and between 8:00 and 9:00 AM in the Medical Diagnostic Laboratory of Mashhad City, Iran.

The serum level of fasting glucose (Glucose kit; Pars Azmoon Co, Tehran, Iran) was measured by glucose oxidase method and the Beckman Glucose Analyzer (Beckman Instruments, Irvine, CA). The insulin was also measured by the Radioimmunoassay (RIA) method and using Nucleo Immune commercial kit (Stillwater, MN). The insulin resistance index was also obtained using HOMA-IR Equation 1 [23]. Also, TC, HDL-C, LDL-C, and TG were measured by the enzymatic method and laboratory Kit of Pars Azmoon Co. 
Equation 1:

HOMA-IR $=$ Fasting insulin $(\mu U / m L) \times$ Fasting glucose $(\mathrm{mg} / \mathrm{L}) / 22.5$

\section{Maximum oxygen consumption}

The maximum oxygen consumption was estimated using a treadmill according to the Naughton protocol. Naughton protocol was performed in 10 two-minute steps. Except for the first stage, conducted at speed of one mile per hour, the steady speed was two miles per hour in all stages. In steps, one and two, the slope of the device was zero, but from the third stage, the slope of the device increased by $3.5 \%$ at each stage. In the Naughton protocol, the maximum oxygen consumption was calculated by Equation 2 as follows:

Equation 2:

Excess maximum oxygen $(\mathrm{mL} / \mathrm{kg} / \mathrm{min})=1.61$ (time to $\mathrm{min}$ ute) +3.6

\section{Aerobic and bariatric exercise programs}

In this study, the regular exercise program included a 12week training, performed three times per week and each session lasted 30 to 60 minutes. This period included 15 minutes of warm-up, 10 to 25 minutes of aerobic exercises and 10 minutes of cool down. The warm-up exercises included strolling, aerobic steps, walking, tensile movements, and general joint warm up. Then, the aerobic exercise program was performed, including walking, jogging, and running at $60-65 \%$ of the heart rate reserve in each patient which was specified in advanced. The first session of aerobic exercise lasted 20 minutes and one minute was added to the aerobic exercise period per session. Thus, from the fourth to the eighth week, the training time was about 30 minutes and from the eighth to the twelfth week it increased to 50 and 60 minutes.

At the end of each training session, cool down and returning to the normal body condition were performed along with jogging, walking, stretching, and relaxation exercises.
The heart rate intensity was maintained in the calculated range by a heart rate watch. The maximum heart rate was calculated using 220-age formula. Exercise intensity was controlled using Polar heart rate monitor (made in Finland). During this period, members of the bariatric training group, inspired by a Table of 6 houses in space or on the ground, implemented the coach exercises and moved the hands and feet on the Table houses. The subjects performed various movements designed by the coach at a specific speed for 90 seconds. Then, the selected movements were changed after 90 seconds.

\section{Statistical analysis}

The obtained data were analyzed using SPSS. The normal distribution of data and homogeneity of variance were verified by the Shapiro-Wilk test and Levene's test, respectively. We used dependent t-test and Analysis of Covariance (ANCOVA) to compare intra- and intergroup variations, respectively. $\mathrm{P}<0.05$ was considered as the level of significance.

\section{Results}

The subjects' characteristics in the aerobic exercise and bariatric groups are listed in Table 1. According to Table 2, in both aerobic and bariatric groups, the dependent t-test results suggested a significant decrease in weight, BMI, serum glucose levels, insulin, and insulin resistance index. Only in the aerobic exercise group, the concentration of HDL, low-density lipoprotein, and total cholesterol significantly increased and decreased, respectively.

In both exercise groups, the levels of triglyceride significantly decreased. Moreover, the maximum oxygen consumption significantly increased only in the aerobic exercise group. In addition, according to Table 2, the average changes in weight, BMI, insulin, insulin resistance index, HDL and maximum oxygen consumption significantly differed between the aerobic and bariatric groups.

Table 1. The subjects' demographic characteristics

\begin{tabular}{|c|c|c|c|c|}
\hline \multirow{2}{*}{ Groups } & \multicolumn{4}{|c|}{ Variations (Mean $\pm S D$ ) } \\
\hline & Age, y & Height, M & Weight, kg & $\mathrm{BMI}, \mathrm{kg} / \mathrm{m}^{2}$ \\
\hline Aerobic $(n=12)$ & $40.50 \pm 4.46$ & $1.56 \pm 0.531$ & $76.48 \pm 3.41$ & $31.39 \pm 2.53$ \\
\hline Bariatric $(n=12)$ & $41.50 \pm 3.87$ & $1.59 \pm 0.550$ & $77.60 \pm 5.66$ & $30.75 \pm 3.05$ \\
\hline
\end{tabular}


Table 2. Changes in insulin resistance index, lipid profile, and maximum rate of oxygen consumption in women with knee osteoarthritis

\begin{tabular}{|c|c|c|c|c|c|c|c|}
\hline \multirow{3}{*}{ Variables } & \multirow{3}{*}{ Group } & \multicolumn{2}{|c|}{ Stages } & \multicolumn{4}{|c|}{ Variations } \\
\hline & & & & \multirow{2}{*}{$\mathbf{T}$} & \multirow{2}{*}{$\mathrm{P}^{*}$} & \multirow{2}{*}{$\mathbf{F}$} & \multirow{2}{*}{$\mathrm{P} * *$} \\
\hline & & Pre-test & Post-test & & & & \\
\hline \multirow{2}{*}{$\begin{array}{c}\text { Weight, } \\
\text { kg }\end{array}$} & Aerobic & $76.48 \pm 3.41$ & $75.64 \pm 3.32$ & 15.91 & $0.001+$ & \multirow{2}{*}{3.45} & \multirow{2}{*}{$0.002+$} \\
\hline & Bariatric & $77.60 \pm 5.66$ & $77.27 \pm 5.64$ & 2.42 & $0.03+$ & & \\
\hline \multirow{2}{*}{$\begin{array}{c}\mathrm{BMI}, \\
\mathrm{kg} / \mathrm{m}^{2}\end{array}$} & Aerobic & $31.39 \pm 2.53$ & $31.04 \pm 2.49$ & 14.79 & $0.001+$ & \multirow{2}{*}{3.19} & \multirow{2}{*}{$0.004^{\dagger}$} \\
\hline & Bariatric & $30.75 \pm 3.05$ & $30.61 \pm 2.94$ & 2.31 & $0.04+$ & & \\
\hline \multirow{2}{*}{$\begin{array}{l}\mathrm{FBS}, \\
\mathrm{mg} / \mathrm{dL}\end{array}$} & Aerobic & $95.58 \pm 4.35$ & $91.41 \pm 3.20$ & 4.05 & $0.002+$ & \multirow{2}{*}{0.00} & \multirow{2}{*}{1.00} \\
\hline & Bariatric & $94.25 \pm 5.67$ & $90.08 \pm 5.72$ & 2.49 & $0.03+$ & & \\
\hline \multirow{2}{*}{$\begin{array}{l}\text { Insulin, } \\
\mathrm{IU} / \mathrm{mL}\end{array}$} & Aerobic & $18.32 \pm 2.61$ & $15.21 \pm 3.07$ & 7.94 & $0.001+$ & \multirow{2}{*}{3.48} & \multirow{2}{*}{$0.002+$} \\
\hline & Bariatric & $18.34 \pm 2.22$ & $16.90 \pm 2.24$ & 5.18 & $0.001+$ & & \\
\hline \multirow{3}{*}{ Insulin resistance } & Aerobic & $77.80 \pm 11.59$ & $61.93 \pm 13.51$ & 11.11 & $0.001+$ & \multirow{3}{*}{2.74} & \multirow{3}{*}{$0.01+$} \\
\hline & & & & & & & \\
\hline & Bariatric & $77.10 \pm 12.27$ & $67.67 \pm 10.08$ & 5.06 & $0.001^{\dagger}$ & & \\
\hline \multirow{2}{*}{$\begin{array}{l}\mathrm{HDL}-\mathrm{C}, \\
\mathrm{mg} / \mathrm{dL}\end{array}$} & Aerobic & $49.33 \pm 6.86$ & $59.25 \pm 6.12$ & -5.34 & $0.001+$ & \multirow{2}{*}{-2.13} & \multirow{2}{*}{$0.04+$} \\
\hline & Bariatric & $46.75 \pm 6.60$ & $50.08 \pm 4.85$ & 2.08 & 0.20 & & \\
\hline \multirow{2}{*}{$\begin{array}{l}\text { LDL-C, } \\
\mathrm{mg} / \mathrm{dL}\end{array}$} & Aerobic & $110.9 \pm 2.80$ & $101.08 \pm 2.31$ & 2.51 & $0.02+$ & \multirow{2}{*}{1.27} & \multirow{2}{*}{0.21} \\
\hline & Bariatric & $117.3 \pm 1.40$ & $114.17 \pm 1.30$ & 0.91 & 0.38 & & \\
\hline \multirow{2}{*}{$\begin{array}{c}\mathrm{TG}, \\
\mathrm{mg} / \mathrm{dL}\end{array}$} & Aerobic & $174.4 \pm 7.04$ & $145.25 \pm 5.68$ & 2.78 & $0.01+$ & \multirow{2}{*}{1.46} & \multirow{2}{*}{0.15} \\
\hline & Bariatric & $132.5 \pm 3.49$ & $119.8 \pm 3.35$ & 3.14 & $0.001+$ & & \\
\hline \multirow{2}{*}{$\begin{array}{c}\mathrm{TC} \\
\mathrm{mg} / \mathrm{dL}\end{array}$} & Aerobic & $180.5 \pm 2.28$ & $168.9 \pm 2.52$ & 2.52 & $0.02+$ & & \\
\hline & Bariatric & $179.08 \pm 2.61$ & $179.7 \pm 1.98$ & -0.13 & 0.89 & & \\
\hline $\mathrm{VO} 2 \mathrm{~m}$ & Aerobic & $21.41 \pm 2.93$ & $24.25 \pm 2.05$ & 1.19 & $0.008^{+}$ & & \\
\hline $\mathrm{mL} / \mathrm{kg} / \mathrm{min}$ & Bariatric & $20.91 \pm 2.83$ & $21.08 \pm 3.10$ & 2.22 & 0.88 & 1.J & 0.000 \\
\hline
\end{tabular}

† Significance level: $\mathrm{P}<0.05$

* $\mathrm{P}$ of within-group comparison

PHYSICAL TREA $\mid$ MENTS

** $\mathrm{P}$ of between-group comparison

\section{Discussion}

The present study compared the effects of 12 weeks of bariatric and aerobic exercises on metabolic syndrome and maximum oxygen consumption indices in women with knee osteoarthritis. Based on the obtained data, 12 weeks of aerobic and bariatric exercises resulted in a significant decrease in serum glucose, insulin, and insulin resistance index. These results are consistent with the findings of Mo- tahari et al. and Le et al. [24, 25]. However, they are inconsistent with the study of Medeiros et al. [26].

Motahari et al. examined the effect of an 8-week aerobic exercise program, performed in three sessions of $30 \mathrm{~min}-$ utes per week with the intensity of $60 \%$ of maximal oxygen consumption in 53 women [25]. They concluded that aerobic exercise decreased plasma glucose, insulin, and insulin resistance index. 
Le et al. reported that implementation of 8-month and 6-week aerobic exercises for 30 to 60 minutes with an intensity of $60-75 \%$ of maximal oxygen consumption in every exercise led to an $18.9 \%$ decrease in insulin resistance in postmenopausal women aged 52-65 years [24]. Medeiros et al. reported that the simultaneous implementation of aerobic exercises with an intensity of $50-75 \%$ of maximal oxygen consumption and the strength exercises with an intensity of 50 to $75 \%$ of repetition maximum increased insulin resistance in 25 obese volunteers [26].

Some of the mechanisms that can increase insulin function after aerobic exercises include increasing post-receptor insulin, increasing the expression of GULT4 glucose transporter protein, increasing glycogen synthase and hexokinase activity, reducing release and enhancing free fatty acid clearance, increasing glucose release from blood to muscles due to increased muscle capillaries and changes in muscle composition to increase glucose uptake [27]. Therefore, aerobic exercises are useful for reducing insulin resistance as well as the risk of type 2 diabetes, especially in obese individuals. Insulin resistance may potentially be mediated by the change in the function of several peptide-mediated secretions of adipocytes, including the tumor necrosis factor alpha, leptin, and adiponectin.

Under the non-inflammatory condition, the Tumor Necrosis Factor alpha (TNF- $\alpha$ )-induced is derived from adipose tissue and its plasma levels are related to body fat. The TNF- $\alpha$-induced contrasts with the insulin messaging, demonstrated by signal reduction through serine phosphorylation. The adiponectin secretion has an inverse relationship with BMI-related features in adipocytes. Adiponectin is a potential inhibitor of the TNF- $\alpha$. Serum levels of adiponectin in obese persons decrease insulin resistance, diabetes mellitus, and metabolic syndrome [14, 28]. Another reason for such contradictory findings may be the differences in duration, intensity, and level of performing the exercise in the subjects.

Based on the obtained results, only in the aerobic exercise group, the maximum oxygen consumption significantly increased. These results are consistent with the findings of Motamedi et al. [29]. However, they are in contrast with the findings of Gelecek et al. [30]. Motamedi et al. studied the effect of eight weeks of aerobic exercise on the maximum oxygen consumption in inactive obese men. They concluded that aerobic exercise significantly increased the maximum oxygen consumption [29].

Gelecek et al. reported that 12 weeks of resistance exercises had no significant impact on the maximum oxygen consumption in 45 postmenopausal women [30]. In other words, regular physical exercises, especially aerobic exercises can reduce the body composition and increase the cardiovascular function of the body. Increased cardiorespiratory fitness through aerobic exercise can be due to the increased number and size of mitochondria, increased enzymes supplied by aerobic energy system, increased levels of oxidation of lipids, and increased levels of oxidation facilitating enzymes [31].

Aerobic exercises cause adaptations such as increasing muscle oxidative capacity (increasing the number of capillaries of muscle fibers), increasing total hemoglobin, reducing glycolysis, increasing end-diastolic volume and increasing the volume of stroke. In addition, it also increases the difference between arterial-venous blood oxygen, Krebs cycle activity, and electron transfer [32]. In addition to regulating key enzymes, citrate synthase regulation occurs in the metabolic pathway of energy production. Citrate synthase is usually considered as a marker for measuring aerobic capacity and mitochondrial density in skeletal muscle and could be improved by aerobic exercise.

Based on the present study, 12 weeks of aerobic and bariatric exercises significantly increase high-density lipoprotein. However, these programs had no significant effects on low-density lipoprotein, total cholesterol, and triglyceride. These results are consistent with the findings of Freitas et al. and Contrò et al. [33, 34]. However, it is inconsistent with the findings of Olson and associates [35]. Freitas et al. examined the effect of 14 weeks of endurance and resistance exercises on the lipid profiles of 49 young men [34]. They concluded that total cholesterol level and triglyceride level decreased significantly. Contrò et al. reported that 12 weeks of circular aerobic exercises and trampoline exercises led to significant decrease in body weight, body fat percentage, and lowdensity lipoprotein in women aged 20 to 50 years [33].

Olson et al. stated that exercising does not change the lipid profiles of people with normal TG, and most of these indices change in individuals with lower TG and LDL-C or lower HDL-C [35]. Participating in aerobic exercises significantly increases the activation of the lipoprotein lipase enzyme and lysine cholesterol acetyltransferase enzyme. These enzymes reduce low-density lipoprotein, triglycerides, and cholesterol, and increases lipoprotein levels [28].

Moderate-intensity exercises increase muscle's demand for energy consumption through lipid metabolism, which results in more oxidation of fat [36]. In this regard, performing aerobic exercises increases beta-adrenergic stimulation, decreases insulin levels, and increases lipolysis rate by three times [37]. The present research had certain limita- 
tions such as divergent nutritional diets and adaptation responses, a limited number of volunteers (as some people refused to participate in the study), and individual differences. Therefore, cautions must be taken in interpreting and generalizing the results.

The aerobic and bariatric exercises can modulate the index of insulin resistance, maximum oxygen consumption and lipid profile in women with knee osteoarthritis. Therefore, it is recommended that both types of exercises be used to prevent the adverse effects of cardiovascular diseases. They can also be considered as an essential part of the patient's lifestyle. However, further research is required to examine the changes in markers of insulin resistance and lipid profile in women with knee osteoarthritis, in different time periods, and different exercise intensities.

\section{Ethical Considerations}

\section{Compliance with ethical guidelines}

The present study was approved by the Ethics Committee of the Faculty of Humanities and Basic Sciences of Bojnourd Branch, Islamic Azad University (Code: 478521).

\section{Funding}

This work was financially supported by Bojnord Branch, Islamic Azad University.

\section{Authors contributions}

All authors contributed in preparing this article.

\section{Conflict of interest}

The authors declare no conflict of interest.

\section{References}

[1] Cooper JA. Factors affecting circulating levels of peptide YY in humans: A comprehensive review. Nutrition Research Reviews. 2014; 27(1):186-97. [DOI:10.1017/S0954422414000109] [PMID]

[2] Loeser RF, Goldring SR, Scanzello CR, Goldring MB. Osteoarthritis: A disease of the joint as an organ. Arthritis \& Rheumatism 2012; 64(6):1697-707. [DOI:10.1002/art.34453] [PMID] [PMCID]

[3] Cooper C, McAlindon T, Coggon D, Egger P, Dieppe P. Occupational activity and osteoarthritis of the knee. Annals of the Rheumatic Diseases. 1994; 53(2):90-3. [DOI:10.1136/ard.53.2.90] [PMID] [PMCID]
[4] Cecil RL, Wyngaarden JB, Smith LH, Bennett JC. Cecil textbook of medicine. Philadelphia: Saunders; 1994.

[5] Järvholm B, Lewold S, Malchau H, Vingård E. Age, bodyweight, smoking habits and the risk of severe osteoarthritis in the hip and knee in men. European Journal of Epidemiology. 2005; 20(6):537-42. [DOI:10.1007/s10654-005-4263-x] [PMID]

[6] Kluzek S, Newton JL, Arden NK. Is osteoarthritis a metabolic disorder? British Medical Bulletin. 2015; 115(1):111-21. [DOI:10.1093/bmb/ldv028] [PMID]

[7] Li H, George DM, Jaarsma RL, Mao X. Metabolic syndrome and components exacerbate osteoarthritis symptoms of pain depression and reduced knee function. Annals of Translational Medicine. 2016; 4(7):133. [PMID] [PMCID]

[8] Romeo GR, Lee J, Shoelson SE. Metabolic syndrome, insulin resistance, and roles of inflammation-mechanisms and therapeutic targets. Arteriosclerosis, Thrombosis, and Vascular Biology. 2012; 32(8):1771-6. [DOI:10.1161/ATVBAHA.111.241869] [PMID] [PMCID]

[9] Reaven GM. Pathophysiology of insulin resistance in human disease. Physiological Reviews. 1995; 75(3):473-86. [DOI:10.1152/ physrev.1995.75.3.473] [PMID]

[10] Ford ES, Giles WH, Mokdad AH. Increasing prevalence of the metabolic syndrome among US adults. Diabetes Care. 2004; 27(10):2444-9. [DOI:10.2337/diacare.27.10.2444] [PMID]

[11] Atashak S, Batourak K, Azizbeigi K. [The effect of moderateintensity aerobic exercise training on metabolic syndrome factors and acylated ghrelin in middle-age women (Persian)]. Razi Journal of Medical Sciences. 2017; 24(159):10-20.

[12] Manson JE, Skerrett PJ, Greenland P, VanItallie TB. The escalating pandemics of obesity and sedentary lifestyle: A call to action for clinicians. Archives of Internal Medicine. 2004; 164(3):24958. [DOI:10.1001/archinte.164.3.249] [PMID]

[13] Bonakdaran S, Barazandeh Ahmadabadi F. Assessment of insulin resistance in idiopathic hirsutism in comparison with Polycystic Ovary Syndrome (PCOS) patients and healthy individuals. Medical Journal of Mashhad University of Medical Sciences. 2014; 56(6):340-6.

[14] Attarzadeh Hosseini SR, Mir E, Hejazi K, Mir Sayeedi M. [The effect of eight weeks combined training on some insulin resistance markers in middle-aged men (Persian)]. Medical Journal of Mashhad University of Medical Sciences. 2015; 58(3):129-36.

[15] Nadeem M, Ahmed SS, Mansoor S, Farooq S. Risk factors for coronary heart disease in patients below 45 years of age. Pakistan Journal of Medical Sciences. 2013; 29(1):91-6. [DOI:10.12669/pjms.291.2828] [PMID] [PMCID]

[16] Pyöraalä M, Miettinen H, Laakso M, Pyöralä K. Hyperinsulinemia predicts coronary heart disease risk in healthy middleaged men: The 22-year follow-up results of the Helsinki policemen study. Circulation. 1998; 98(5):398-404. [DOI:10.1161/01. CIR.98.5.398] [PMID]

[17] Gray SR, Baker G, Wright A, Fitzsimons CF, Mutrie N, Nimmo MA. Scottish physical activity research collaboration. The effect of a 12 week walking intervention on markers of insulin resistance and systemic inflammation. Preventive Medicine. 2009; 48(1):39-44. [DOI:10.1016/j.ypmed.2008.10.013] [PMID]

[18] Carrel AL, McVean JJ, Clark RR, Peterson SE, Eickhoff JC, Allen DB. School-based exercise improves fitness, body composition, 
insulin sensitivity, and markers of inflammation in non-obese children. Journal of Pediatric Endocrinology and Metabolism. 2009; 22(5):409-16. [DOI:10.1515/JPEM.2009.22.5.409] [PMID]

[19] Bruno E, Roveda E, Vitale J, Montaruli A, Berrino F, Villarini A, et al. Effect of aerobic exercise intervention on markers of insulin resistance in breast cancer women. European Journal of Cancer Care. 2018; 27(2):e12617. [DOI:10.1111/ecc.12617] [PMID]

[20] Lin CH, Ho CW, Chen LC, Chang CC, Wang YW, Chiou CP, et al. Effects of a 12-week exercise training on insulin sensitivity, quality of life, and depression status in patients with type 2 diabetes. Journal of Medical Sciences. 2017; 37(6):227-36. [DOI:10.4103/jmedsci.jmedsci_68_17]

[21] Reginster JY. The prevalence and burden of arthritis. Rheumatology. 2002; 41(suppl-1):3-6. [DOI:10.1093/rheumatology/41. S1.3] [PMID]

[22] Felson DT. Epidemiology of hip and knee osteoarthritis. Epidemiologic Reviews. 1988; 10:1-28. [DOI:10.1093/oxfordjournals. epirev.a036019] [PMID]

[23] Sâmpelean D, Hănescu BI, Han AN, Adam M, Casoinic F. The prognosis of glycoregulation disturbances and insulin secretion in alcoholic and $\mathrm{C}$ virus liver cirrhosis. Romanian Journal of Internal Medicine. 2009; 47(4):387-92. [PMID]

[24] Le S, Mao L, Lu D, Yang Y, Tan X, Wiklund P, et al. Effect of aerobic exercise on insulin resistance and central adiposity disappeared after the discontinuation of intervention in overweight women. Journal of Sport and Health Science. 2016; 5(2):166-70. [DOI:10.1016/j.jshs.2016.04.003] [PMID] [PMCID]

[25] Motahari-Tabari N, Shirvani MA, Shirzad-e-Ahoodashty M, Yousefi-Abdolmaleki E, Teimourzadeh M. The effect of 8 weeks aerobic exercise on insulin resistance in type 2 diabetes: A randomized clinical trial. Global Journal of Health Science. 2015; 7(1):115-21. [PMID] [PMCID]

[26] Medeiros ND, de Abreu FG, Colato AS, de Lemos LS, Ramis TR, Dorneles GP, et al. Effects of concurrent training on oxidative stress and insulin resistance in obese individuals. Oxidative Medicine and Cellular Longevity. 2015; 2015:697181. [DOI:10.1155/2015/697181]

[27] Eriksson J, Taimela S, Eriksson K, Parviainen S, Peltonen J, Kujala U. Resistance training in the treatment of non-insulin-dependent diabetes mellitus. International Journal of Sports Medicine. 1997; 18(4):242-6. [DOI:10.1055/s-2007-972627] [PMID]

[28] Das AK, Leggett RE, Whitbeck C, Eagen G, Levin RM. Effect of doxazosin on rat urinary bladder function after partial outlet obstruction. Neurourology and Urodynamics-International Continence Society. 2002; 21(2):160-6. [DOI:10.1002/ nau.10045] [PMID]

[29] Motamedi P, Nikroo H, Hejazi K. The effects of eight-weeks aerobic training on serum leptin levels, anthropometric indices and VO2max in sedentary obese men. Journal of Ergonomics. 2017; 5(1):36-42. [DOI:10.21859/joe-05015]

[30] Gelecek N, Ilçin N, Subaşi SS, Acar S, Demir N, Ormen M. The effects of resistance training on cardiovascular disease risk factors in postmenopausal women: A randomized-controlled trial. Health Care for Women International. 2012; 33(12):107285. [DOI:10.1080/07399332.2011.645960] [PMID]

[31] Nikroo H, Nematy M, Sima HR, Attarzade HS. The effect of restricted diet with or without aerobic training program on cardio respiratory fitness and anthropometric indices in patients with non alcoholic steatohepatitis. Journal of North Khorasan University of Medical Sciences. 2011; 3(3):91-9. [DOI:10.29252/ jnkums.3.3.91]

[32] Duncan GE, Perri MG, Anton SD, Limacher MC, Martin AD, Lowenthal DT, et al. Effects of exercise on emerging and traditional cardiovascular risk factors. Preventive Medicine. 2004; 39(5):894-902. [DOI:10.1016/j.ypmed.2004.03.012] [PMID]

[33] Contrò V, Bianco A, Cooper J, Sacco A, Macchiarella A, Traina $\mathrm{M}$, et al. Effects of different circuit training protocols on body mass, fat mass and blood parameters in overweight adults. Journal of Biological Research. 2017; 90(1). [DOI:10.4081/ jbr.2017.6279]

[34] Freitas A, Costa A, Pereira C, Batalha N. Effects of endurance versus strength training programs in the lipid profile of sedentary young adults. Paper presented at: Proceedings of the International Congress of the Research Center in Sports Sciences, Health Sciences \& Human Development. 11-12 November 2016; Évora, Portugal.

[35] Olson TP, Dengel DR, Leon AS, Schmitz KH. Changes in inflammatory biomarkers following one-year of moderate resistance training in overweight women. International Journal of Obesity. 2007; 31(6):996-1003. [DOI:10.1038/ sj.ijo.0803534] [PMID]

[36] Romijn JA, Coyle EF, Sidossis LS, Rosenblatt J, Wolfe RR. Substrate metabolism during different exercise intensities in endurance-trained women. Journal of Applied Physiology. 2000; 88(5):1707-14. [DOI:10.1152/jappl.2000.88.5.1707] [PMID]

[37] Wolfe RR, Klein SA, Carraro FA, Weber JM. Role of triglyceride-fatty acid cycle in controlling fat metabolism in humans during and after exercise. American Journal of Physiology-Endocrinology and Metabolism. 1990; 258(2):E382-9. [DOI:10.1152/ ajpendo.1990.258.2.E382] [PMID] 